\title{
Astrophysics at n_TOF facility
}

G.Tagliente $^{1}$, U.Abbondanno ${ }^{2}$, G.Aerts ${ }^{3}$, H.Alvarez ${ }^{4}$, F.Alvarez-Velarde ${ }^{5}$, S.Andriamonje ${ }^{3}$, J.Andrzejewski ${ }^{6}$, P.Assimakopoulos ${ }^{7} \dagger$, L.Audouin ${ }^{8}$, G.Badurek ${ }^{9}$, P.Baumann ${ }^{10}$, F.Bečvári ${ }^{11}$, F.Belloni ${ }^{2}$, E.Berthoumieux ${ }^{3}$, S.Bisterzo ${ }^{12}$, F.Calviñ ${ }^{13}{ }^{2}$, M.Calviani ${ }^{14}$, D.Cano-Ott ${ }^{5}$, R.Capote ${ }^{15,16}$, C.Carrapiço $^{17}$, P.Cennini ${ }^{18}$, V.Chepe ${ }^{19}$, E.Chiaveri ${ }^{18}$, N.Colonna ${ }^{1}$, G.Cortes ${ }^{13}$, A.Couture $^{20}$, J.Cox ${ }^{20}$, M.Dahlfors ${ }^{18}$, S.David ${ }^{10}$, I.Dillman ${ }^{8}$, C.Domingo-Pardo ${ }^{21}$, W.Dridi ${ }^{3}$, I.Duran $^{4}$, C.Eleftheriadis ${ }^{22}$, M.Embid-Segura ${ }^{5}$, L.Ferrant ${ }^{23} \uparrow$, A.Ferrari ${ }^{18}$, R.FerreiraMarques $^{19}$, K.Fujii ${ }^{2}$, W.Furman ${ }^{24}$, R.Gallino ${ }^{12}$, I.Goncalves ${ }^{19}$, E.Gonzalez-Romero ${ }^{5}$,

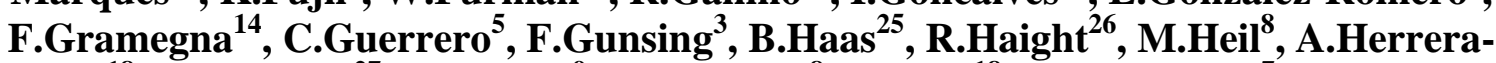
Martinez $^{18}$, M.Igashira ${ }^{27}$, E.Jericha ${ }^{9}$, F.Käppeler ${ }^{8}$, Y.Kadi ${ }^{18}$, D.Karadimos ${ }^{7}$, D.Karamanis ${ }^{7}$, M.Kerveno ${ }^{10}$, P.Koehler ${ }^{28}$, E.Kossionides ${ }^{29}$, M.Krtička ${ }^{11}$, C.Lamboudis ${ }^{22}$, H.Leeb', A.Lindote $^{19}$, I.Lopes ${ }^{19}$, M.Lozano ${ }^{16}$, S.Lukic ${ }^{10}$, J.Marganiec $^{6}$, S.Marrone ${ }^{1}$, T.Martinez,

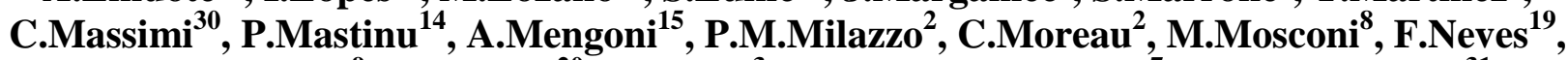
H.Oberhummer', S.O'Brien ${ }^{20}$, J.Pancin ${ }^{3}$, C.Papachristodoulou ${ }^{7}$, C.Papadopoulos ${ }^{31}$,

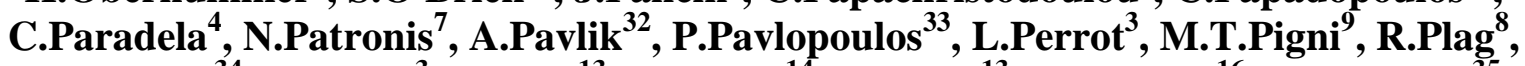
A.Plompen $^{34}$, A.Plukis ${ }^{3}$, A.Poch ${ }^{13}$, J.Praena ${ }^{14}$, C.Pretel $^{13}$, J.Quesada ${ }^{16}$, T.Rauscher ${ }^{35}$, R.Reifarth $^{26}$, C.Rubbia ${ }^{36}$, G.Rudolf ${ }^{10}$, P.Rullhusen ${ }^{34}$, J.Salgado $^{17}$, C.Santos ${ }^{17}$, L.Sarchiapone ${ }^{18}$, I.Savvidis $^{22}$, C.Stephan ${ }^{23}$, J.L.Tain ${ }^{21}$, L.Tassan-Got ${ }^{23}$, L.Tavora ${ }^{17}$, R.Terlizzi ${ }^{1}$, G.Vannini ${ }^{30}$,

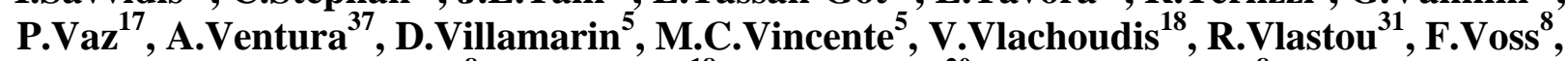
S.Walter ${ }^{8}$, H.Wendler ${ }^{18}$, M.Wiescher ${ }^{20}$ and K.Wisshak ${ }^{8}$

${ }^{1}$ INFN, Trieste, Italy; ${ }^{2}$ INFN, Bari, Italy; ${ }^{3}$ CEA/Saclay, Gif-sur-Yvette, France; ${ }^{4}$ Univ. Santiago de Compostela, Spain; ${ }^{5}$ CIEMAT, Madrid, Spain; ${ }^{6}$ Univ. Lodz, Poland; ${ }^{7}$ Univ. Ioannina, Greece; ${ }^{8}$ FZK, Institut für Kernphysik, Germany; ${ }^{9}$ Technische Universität Wien, Austria; ${ }^{10}$ CNRS/IN2P3 - IReS, Strasbourg, France; ${ }^{11}$ Univ. Prague, Czech Republic; ${ }^{12}$ Dip. Fisica Generale, Univ. Torino, Italy; ${ }^{13}$ Univ. Politecnica Catalunya, Barcelona, Spain; ${ }^{14}$ INFN, Laboratori Nazionali di Legnaro, Italy; ${ }^{15}$ IAEA, Vienna, Austria;

${ }^{16}$ Univ. Sevilla, Spain; ${ }^{17}$ ITN, Lisbon, Portugal; ${ }^{18}$ CERN, Geneva, Switzerland; ${ }^{19}$ LIP - Coimbra \& Dep.Fisica Univ. Coimbra, Portugal; ${ }^{20}$ Univ. Notre Dame, USA $;{ }^{21}$ Inst.Fisica Corpuscular, CSIC-Univ. Valencia, Spain; ${ }^{22}$ Aristotle Univ. Thessaloniki, Greece; ${ }^{23}$ CNR S/IN2P3 - IPN, Orsay, France; ${ }^{24} J I N R$, Frank Lab. Neutron Physics, Dubna, Russia; ${ }^{25}$ CNRS/IN2P3 - IPN, Orsay, France; ${ }^{26}$ LANL, USA $;{ }^{27}$ Tokyo Inst. Technology, Japan; ${ }^{28}$ ORNL, Physics Division, USA $;{ }^{29}$ NCSR, Athens, Greece; ${ }^{30}$ Dip. Fisica, Univ. Bologna, \& INFN, Bologna, Italy; ${ }^{31}$ National Technical Univ. Athens, Greece; ${ }^{32}$ Inst. für Fakultät für Physik, Univ. Wien, Austria; ${ }^{33}$ Pôle Univ. L. de Vinci, Paris, France; ${ }^{34}$ CEC-JRC-IRMM, Geel, Belgium;

${ }^{35}$ Dep. Physics and Astronomy, Univ. Basel, Switzerland; ${ }^{36}$ Univ. Pavia,Italy; ${ }^{37}$ ENEA, Bologna, Italy. The n_TOF Collaboration + (deceased)

The neutron time of flight (n_TOF) facility at CERN is a neutron spallation source, its white neutron energy spectrum ranges from thermal to several $\mathrm{GeV}$, covering the full energy range of interest for nuclear astrophysics, in particular for measurements of the neutron capture crtoss section required in s-process nucleosynthesis. This contribution gives an overview on the astrophysical program made at $\mathrm{n}_{-}$TOF facility, the results and the implications will be reported.

\section{Stellar nucleosyntesis}

The origin of the elements is an important topic to understand the evolution of the universe. Hydrogen and helium, and small amounts of lithium, were formed in the period between about 100 seconds and 20 minutes after the big bang [1]. This period of primordial nucleosynthesis was followed by galactic condensation and the formations of the stars. All elements heavier than lithium have been formed in stars, and the elements heavier than iron have been formed via neutron capture processes in the stars. The isotopic abundances in the solar system reflect the average composition of the galaxy as it was $5.5 \times 10^{9}$ years ago. Spectral information of stellar 
environments and isotopic analyses of presolar dust grains provide important observation to validate stellar evolution models.

\subsection{The s-process}

Stellar nucleosynthesis has first been extensively reviewed in the reference work [2] and more recently in [3][4]. The isotopes up to ${ }^{56} \mathrm{Fe}$ can be synthesized by fusion reactions during the different stages of the evolution of a star. It is nowadays well established that neutron capture processes in red giant stars and supernovae are responsible for the formation of nearly all isotopes with higher masses [1][5]. This was first recognized by the discovery of technetium in red giant stars [6].

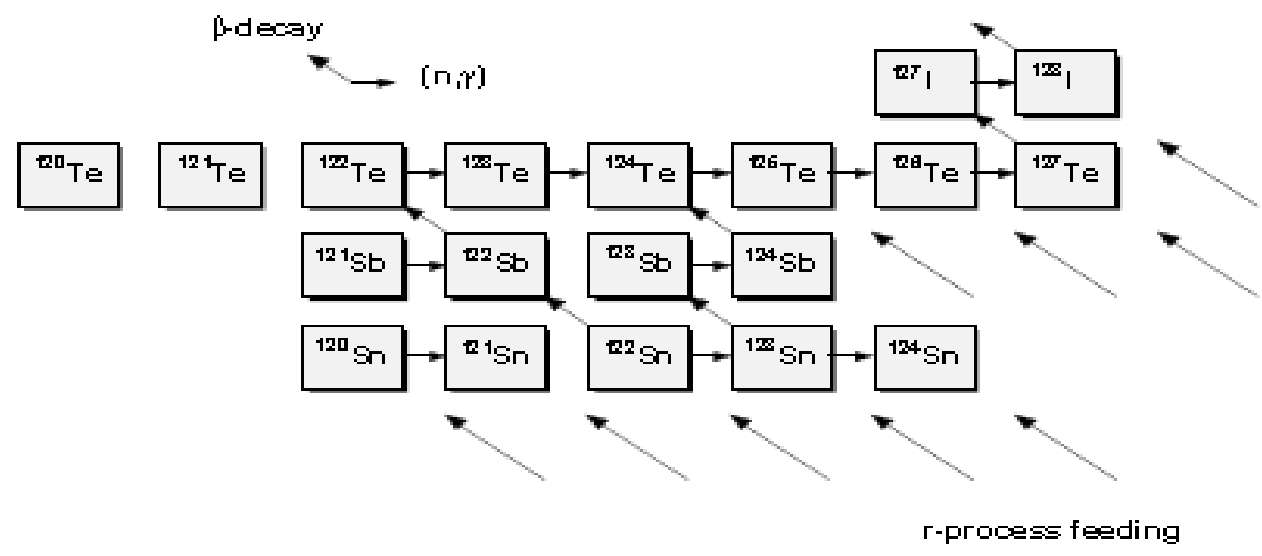

Figure 1.2 s-process path in the $\mathrm{Sn}$ region with s-only and r-only isotopes

The neutron capture mechanisms are known as s- and r-processes, where "s" stands for slow and " $\mathrm{r}$ " for rapid referring to the time scale of the $\beta$-decay. The neutron source are manly the ${ }^{22} \mathrm{Ne}(\alpha, \mathrm{n}){ }^{25} \mathrm{Mg}$ and ${ }^{13} \mathrm{C}(\alpha, \mathrm{n}){ }^{16} \mathrm{O}$ reactions. The s- and $\mathrm{r}$ - processes are important for the stable and neutron reach isotopes. A thorough knowledge of the s-process, for which much more experimental data is available, constrains the possibilities of the r-process. A competing mechanism is the pprocess, referring to photodintegration reaction like $(\gamma, n),(\gamma, p)$ and $(\gamma, \alpha)$, they influence the abundances from the proton rich side.

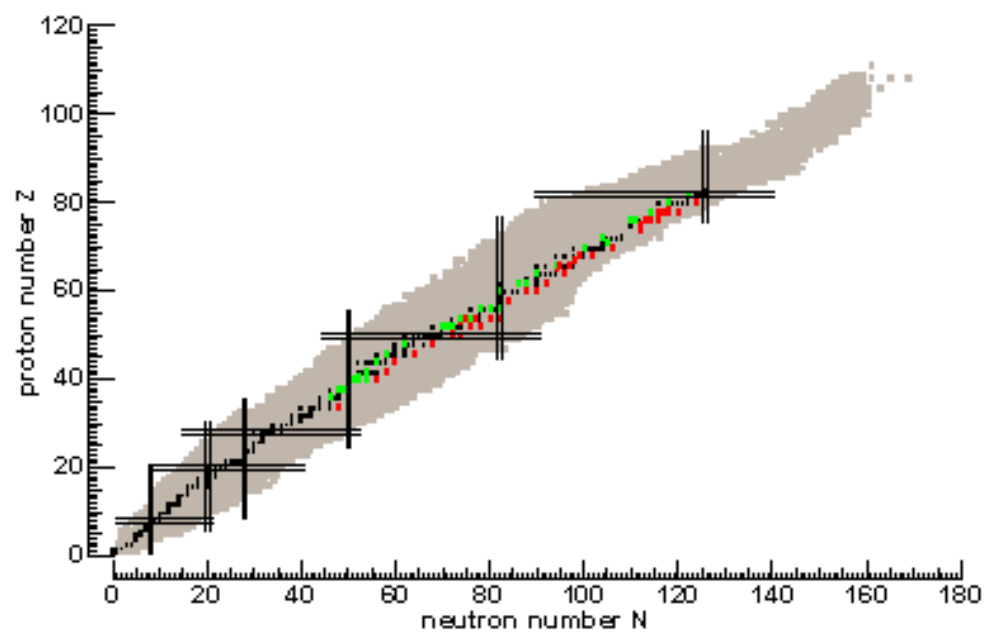

Figure 1.3: The chart of the nuclides showing the stable isotopes along the s-process, in black, and the s-only and r-only isotopes, in green and red respectively 
If the compound nucleus is unstable against the $\beta$-decay, it may decay before it captures a second neutron. This is what happens in the s-process which is the principal process of synthesis in the red giant stars, where thousands of years may pass between two successive neutron captures on a nucleus. In this way many of the isotopes from ${ }^{56} \mathrm{Fe}$ to ${ }^{209} \mathrm{Bi}$ are formed. Heavier nuclei than Bi are unstable and cannot be formed by neutron capture anymore. The s-process path follows closely the valley of stability in the chart of the nuclei and ends at ${ }^{209} \mathrm{Bi}$.

In the r-process the neutron capture process is much faster and occurs on much shorter time scales. The time between consecutive neutron captures is the order of seconds. In order to achieve the according extremely high neutron fluxes, the astrophysical site for the r-process is believed to be of explosive nature, like in a supernova. Neutron capture cross section are a key ingredient in the development of stellar models using the calculation of nuclear abundances in stellar environments. At the branching points uncertainties in the cross sections can propagate into large differences in the production of higher mass nuclei within a given model.

In a very schematic quantitative description of the s-process, starting from the seed nucleus ${ }^{56} \mathrm{Fe}$ and assuming constant temperature and neutron density, for a s-only nucleus the product of the average capture cross section $\left\langle\sigma_{\gamma}\right\rangle_{k T, A}$ and the abundance of the isotope $N_{s, A}$ constant

$$
\left\langle\sigma_{\gamma}\right\rangle_{k T, A} N_{s, A}=\text { constant }
$$

Indeed this is roughly the case, except for the nuclei with magic numbers $(\mathrm{N}=28,50,82,126)$ around $A=88,140$, and 208, which have very low cross sections. These nuclei are bottlenecks in the s-process paths, and show up as abundance peaks. The s-only nuclei are shielded from r-process by stable isobars of nuclei with lower $\mathrm{Z}$ and which contributions from proton rich side of the valley of stability are commonly neglected. In the same way r-only nuclei have no contribution from the sprocess.

In fig 1.3 a simplified part of the s-process is shown in the $\mathrm{Z}=50$ vicinity starting from ${ }^{120} \mathrm{Sn}$. Some stable nuclides, like ${ }^{124} \mathrm{Sn}$ and ${ }^{130} \mathrm{Te}$ are not reached by the s-process put but are shielded from r-process by the nuclei ${ }^{122} \mathrm{Sb},{ }^{123} \mathrm{Sb}$ and ${ }^{124} \mathrm{Sb}$. These are s-only isotopes. The about 30 $\mathrm{s}$ - and about $40 \mathrm{r}$-only isotopes provide a means to distinguish between the two processes. They are given in figure 1.4 showing also the stable nuclides.

\section{The n_TOF facility}

The n_TOF facility, based on an idea by Rubbia at all [7], locate at CERN Geneva Switzerland became fully operational in May 2002, when the scientific program has started. A detailed description can be found in ref [8]. Neutron are produced by spallation reaction induced by a pulsed, $6 \mathrm{~ns}$ wide, $20 \mathrm{GeV} / \mathrm{c}$ proton beam with up to $7 \times 10^{12}$ proton per pulse, impinging on a $80 \times 80 \times 60 \mathrm{~cm}^{3}$ lead target. A $5 \mathrm{~cm}$ water slab surrounds the lead target acting as a coolant and as moderator of the initial fast neutron spectrum. An isolethargic neutron flux distribution is produced over a wide range of energy $(1 \mathrm{eV}-250 \mathrm{MeV})$.

Neutron emerging from the target propagate in the vacuum pipe inside the time-of-flight tunnel $200 \mathrm{~m}$ long. Two collimators are present along the flight path, one of the diameter of $13,5 \mathrm{~cm}$ placed at $135 \mathrm{~m}$ from the lead target and one at $180 \mathrm{~m}$ with a diameter of $2 \mathrm{~cm}$ for the capture measurements. This collimation results in a Gaussian-shaped beam profile [9]. A $1.5 \mathrm{~T}$ sweeping magnet placed at $40 \mathrm{~m}$ upstream of the experimental area is used to deflect outside the beam charged particles travelling along the vacuum pipe. For an efficient background suppression, several concrete and iron walls are placed along the time-of-flight tunnel.

The measuring station is located inside the tunnel, centered at $187.5 \mathrm{~m}$ from the spallation target. 
The neutron beam is monitored up to $1 \mathrm{MeV}$ by a low-mass system, based on thin mylar foil with ${ }^{6} \mathrm{Li}$ deposit places in the beam, surrounded by an array of silicon detectors place outside the beam. The detection by the silicon detectors of the triton and $\alpha$ 's produced in the ${ }^{6} \operatorname{Li}(n, \alpha)$ reaction gives a direct measure of the neutron flux. The small amount of material in the beam ensures a negligible level of scattered neutrons. The scattering chamber is made in carbon fibre to minimize the neutron-induced $\gamma$ background.

Measurements of neutron capture cross-sections in the first stage of the project were performed specifically made $\mathrm{C}_{6} \mathrm{D}_{6}$ detectors, and in the second stage of the measurements a $4 \pi$ calorimeter made of $40 \mathrm{BaF}_{2}$ crystal has been used.

The data acquisition system is based on flash ADCs with sampling rate up to $1 \mathrm{GHz}$ for recording the detector signals during nearly $20 \mathrm{~ms}$ off-line analysis. This generate a high data rate but ensures an almost zero dead-time.

In the first phase of the $n_{-}$TOF project, neutron capture measurements were carried with an array of $\mathrm{C}_{6} \mathrm{D}_{6}$ liquid scintillator cells. These detectors have the advantage of being the less sensitive to scattered neutron. Specifically designed $\mathrm{C}_{6} \mathrm{D}_{6}$ where used at $\mathrm{n}_{-} \mathrm{TOF}$, in order to reduce the neutron sensitivity all the material that could produce a neutron capture in the detector were removed or substituted, all the aluminum part were substituted with carbon fibre [10] and also the support material was minimized, allowing to perform measurement of isotopes with a large scattering to capture ratio.

Due to the small solid angle coverage and the low intrinsic efficiency the $\mathrm{C}_{6} \mathrm{D}_{6}$ detectors, which result in an overall efficiency of $\sim 10 \%$, only one $\gamma$-ray per event is detected from the deexcitation cascade following neutron capture. For an accurate cross-section determination, the efficiency of the set-up has to be made independent on the details of the de-excitation cascade, in particular of the $\gamma$-ray multiplicity.

To this end the pulse height weighting function (PHWF) has been used. It consists in suitably modifying by software the detectors response so that the efficiency $\varepsilon_{\gamma}$ is proportional to the photon energy $E_{\gamma}$. Under these conditions the efficiency for detecting a cascade becomes proportional to the known cascade energy Ec and independent of the actual cascade path.

In the second phase of $n_{-}$TOF project the neutron capture measurements have been performed with Total Absorption Calorimeter (TAC). The design of the n_TOF TAC is based o 42fold segmentation consisting of $15 \mathrm{~cm}$ thick $\mathrm{BaF}_{2}$ crystal in the form of truncated pyramids. Each of the 12 pentagonal and 30 hexagonal crystals extends the same solid angle with respect to the sample centre.

On average the crystals exhibit an average energy resolution of $14 \%$ at $662 \mathrm{keV}$ and an excellent time resolution of about 500 ps.

Due to the low cross-section of most the samples of Astrophysics interest measured at $\mathrm{n}_{-} \mathrm{TOF}$, the $\mathrm{C}_{6} \mathrm{D}_{6}$ were preferred for these measurements since the background due to the in-beam $\gamma$ for those detectors is lower.

\section{Experimental campaign}

The Astrophysics experimental campaign was focus on neutron magic nuclei, which act as bottle neck for the flow of s-process, nuclei with A $<120$, branching points isotopes and isotopes of special interest as the Os as a cosmic clock, in the following the description and results of the measurements.

\section{1 ${ }^{151} \mathrm{Sm}(\mathrm{n}, \gamma)$ cross section measurements}

The ${ }^{151} \mathrm{Sm}$ is a branching point in the s-process path, in particular, this branching is sensitive to the temperature at which the s-process is taking place. The accurate determination of the neutron 
capture cross-section of this isotope can thus provide crucial information on the thermodynamics condition of the AGB stars.

The measurement had been performed with the $\mathrm{C}_{6} \mathrm{D}_{6}$ liquid scintillator. The result obtained at $n \_$TOF is $\langle\sigma(n, \gamma)\rangle=3100 \pm 160 \mathrm{mb}$, a value much larger than previous estimated, all based on model calculation, which ranged from 1500 and $2800 \mathrm{mb}$.

The firm estimate of the capture rate for the first time base on experimental value allowed reaching two important conclusions with respect to the s-process nucleosynthesis in this mass region: i) the classical model, based on the phenomenological study of the s-process fails to produce consistent result of the branching at ${ }^{151} \mathrm{Sm}$ and ${ }^{147} \mathrm{Pm}$, ii) the p-process contribution to the production of ${ }^{152} \mathrm{Gd}$ can amount up $30 \%$ of the solar-system observed abundance [11]

\section{2 ${ }^{90,91,92,93,94,96} \mathrm{Zr}$ and ${ }^{139} \mathrm{La}(\mathrm{n}, \gamma)$ cross section measurements}

The neutron capture measurement of these isotopes has a particular relevance in the nuclear astrophysics; since the $\mathrm{Zr}$ belongs to the first s-process peak in the solar abundance distribution at $\mathrm{N}$ $=50$ while the La belongs to the second s-process peak at $\mathrm{N}=82$. The ${ }^{90} \mathrm{Zr}$ and the ${ }^{139} \mathrm{La}$ are neutron magic and are characterized, like the ${ }^{91,92,93,94} \mathrm{Zr}$, by a low neutron capture cross section and are predominately of s-process origin. The most neutron reach $\mathrm{Zr}$ stable isotope ${ }^{96} \mathrm{Zr}$, is traditionally considered to be an r-only isotope with a small s-process admixture[12][13]. Its abundance is considered to be a strong indicator in the efficiency of the ${ }^{22} \mathrm{Ne}$ neutron source during the He shell burning episodes of thermally pulsing AGB stars. The lanthanum acts as bottleneck between the abundant light n-capture element of the first s-process peak and the heavy elements from $\mathrm{Sm}$ up to $\mathrm{Pb}$ and $\mathrm{Bi}$, it is very important for interpreting the element abundance patterns in very old, metal poor stars. Since the La abundance is completely represented by ${ }^{139}$ La it can be used to distinguish the s-process components from the products of explosive r-process nucleosynthesis, the $\mathrm{s} / \mathrm{r}$ ratio is of utmost importance for the galatical chemical evolution.

The major motivation of these measurements was to reduce the uncertainties to a few percent, as required to improve the stellar s-process model. The results of the $\mathrm{Zr}$ isotopes measurements will be reported in more details in a dedicated proceeding of this conference.

The capture cross section of the ${ }^{139} \mathrm{La}$ in term of resonance parameter was measured in a large energy range from $0.6 \mathrm{eV}$ up to $9 \mathrm{keV}$, these results [14] show sizeable differences with respect to the previous experimental data and allow to extract the related nuclear quantities with improved accuracy, in fig 3.1 is reported the comparison of the Maxwellian Averaged Cross Section (MACS) calculated with $\mathrm{n} \_$TOF data with other experimental results.

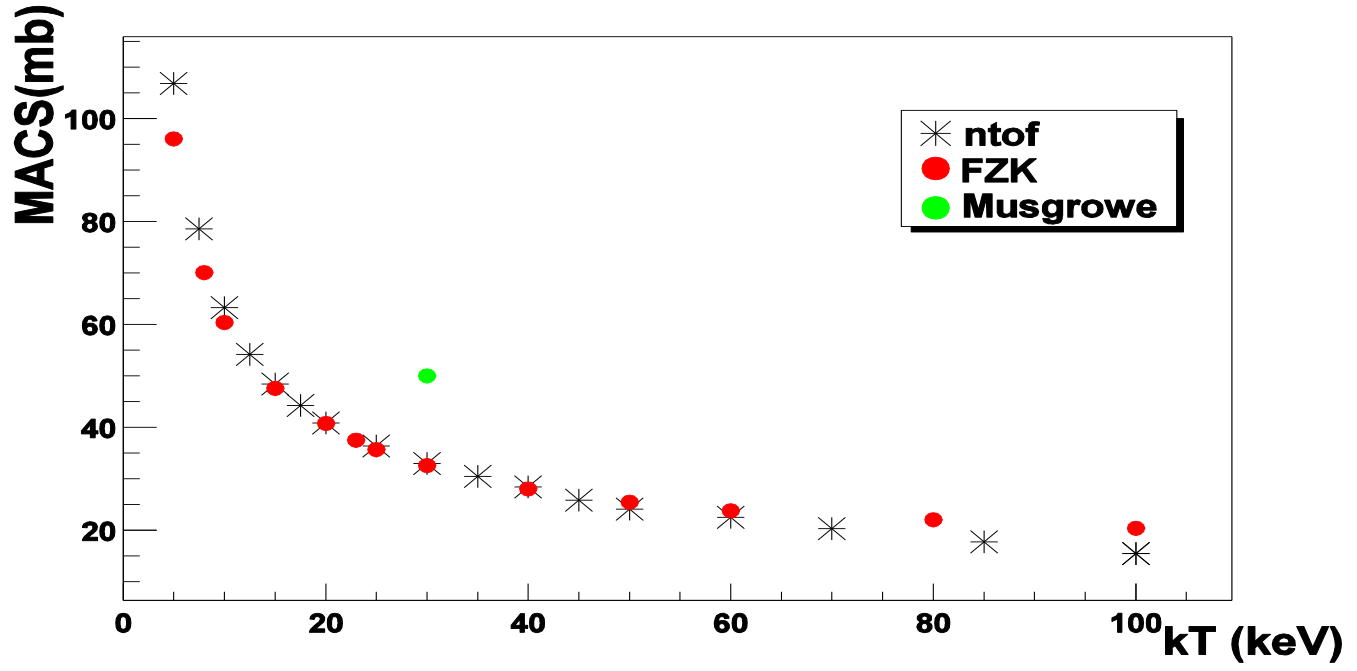

Fig. 3.1 Comparison among the ${ }^{139}$ La MACS calculated with $\mathrm{n}_{-}$TOF data and FZK and Musgrowe data 


\section{$3.3^{204,206,207} \mathrm{~Pb}$ and ${ }^{209} \mathrm{Bi}(\mathrm{n}, \gamma)$ measurements}

The $\mathrm{Pb}$ isotopes and the ${ }^{209} \mathrm{Bi}$ have a special role in the nucleosynthesis, these isotopes represent the termination point of s-process nucleosynthesis, this point is reached since the $\alpha$ recycling of $\mathrm{Po}$ and heavier $\mathrm{Bi}$ isotopes is always faster than further neutron captures. It is important to know the cross section information for the $\mathrm{Pb}$ and $\mathrm{Bi}$ isotopes with very high accuracy in order to determine more exactly the amounts and ratios of these isotopes being produced.

Capture widths and radioactive kernels were determinate in a large range of energy for all isotopes. From these results the MACS have been derived and in many cases large discrepancy were found with values of the previous experiment, in figure 4 is reported the comparison between the ${ }^{204} \mathrm{~Pb}$ MACS calculated with $\mathrm{n}$-TOF and the values reported by Bao [15],

For all isotopes the systematic uncertainties could be improved by a factor two, this allowed to have a firm calculation of the abundances of the s-process component and to constrain the estimation of the r-process component, the results are reported in [16][17][18][19]

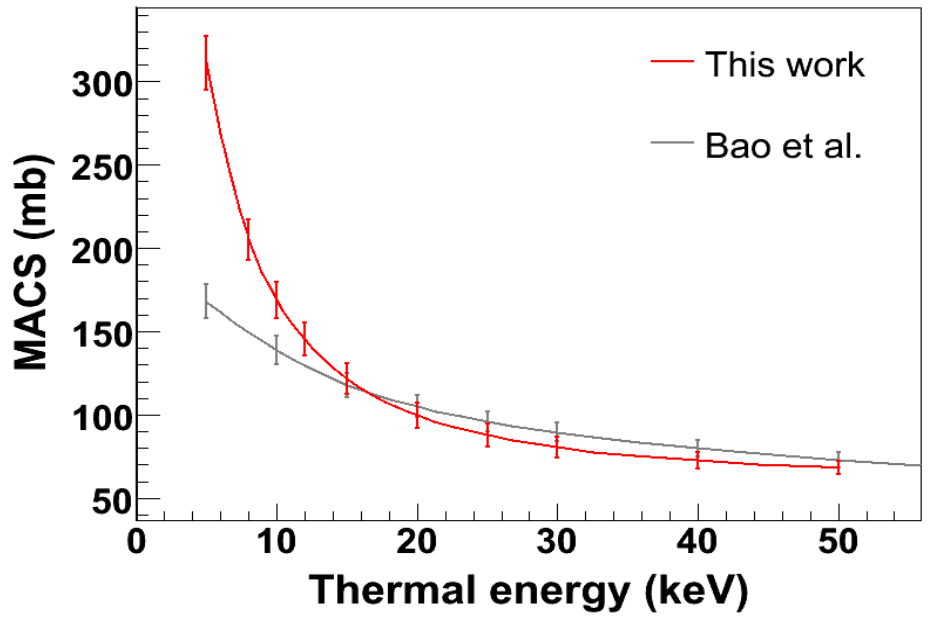

Fig. 4. Comparison between the ${ }^{204} \mathrm{~Pb}$ MACS calculated with n_TOF data and Bao et al.

\section{4 ${ }^{186,187,188}$ Os $(n, \gamma)$ measurements}

The time duration of the nucleosynthesis of the heavy elements produced by neutron capture processes can be used to set limits on the age of the universe [20], among several cosmic clock based on the abundances of long-lived radioactive isotopes the ${ }^{187} \mathrm{Os} /{ }^{187} \mathrm{Re}$ is one of the more interesting.

The clock is based on the extremely long half-life of ${ }^{187} \operatorname{Re}\left(\tau_{1 / 2}=43.3 \mathrm{Gyr}\right)$ decay to ${ }^{187} \mathrm{Os}$ and on the fact that ${ }^{186} \mathrm{Os}$ and ${ }^{187} \mathrm{Os}$ are shielded against direct r-process production. Then, thanks to the well established s-process abundances of the ${ }^{186} \mathrm{Os}$ and ${ }^{187} \mathrm{Os}$, the Re/Os clock can be characterized by the enhancement in the abundance of ${ }^{187}$ Os due to ${ }^{187} \mathrm{Re} \rightarrow{ }^{187}$ Os decay.

\section{REFERENCES}

1. R. A. Alpher, H. A. Bethe, G. Gamow, Phys. Rev. 73,(1948)803.

2. E. M. Burbidge, G. R. Burbidge, W. A. Fowler, F. Hoyle, Synthesis of the elements in stars, Rev. Mod. Phys. 29,(1957)547.

3. G. Wallerstein, et al., Synthesis of the elements in stars: forty years of progress, Rev. Mod. Phys. 62(1999)395.

4. M. Arnould, K. Takahash, Nuclear astrophysics, Rep. Prog. Phys. 62(1999)395.

5. A. G. W. Cameron, Pub. Astr. Soc. Pacific 69(1957)201.

6. P.W. Merrill, Science 115(1952)484. 
7. C. Rubbia et al., Tech. Rep. CERN/LHC/98-02 CERN(1998)

8. U. Abbondanno et al., Tech. Rep. CERN/SL/2002-053 ECT(2003)

9. S. J. Pancin et al., Nucl. Instr. Meth. A 524(2004)102.

10. R. Plag et al., Nucl. Instr. Meth. A 496,(2003)425.

11. U. Abbondanno et al., Phys. Rev. Letters 93,(2004)161103

12. F. Käppeler et al., Prog. Nucl. Part. Phys. 43,(1999)419.

13. K.A. Toukan, K. Debus, F. Käppeler, and G. Reffo, Phys. Rev. C51,(1995)1540.

14. R. Terlizzi et al., Phys Rev. C75,(2007)35807.

15. Z.Y. Bao et al., At. Data Nucl. Data Tables 76,(2000).

16. C. Domingo-Pardo et al., Phys Rev. C75,(2007)15806.

17. C. Domingo-Pardo et al., Phys Rev. C74,(2006)55802.

18. C. Domingo-Pardo et al., Phys Rev. C74,(2006) 25807.

19. C. Domingo-Pardo et al., Phys Rev. C76,(2007)45805.

20. D.D. Clayton et al., Ap.J. 139 (1964) 637 